\title{
Impacto de la ergonomía en la productividad, una revisión sistemática entre los años 2016 - 2021
}

Impact of ergonomics on productivity, a systematic review from 2016 - 2021

Robert Santiago Mamani Hualpa Universidad César Vallejo rsmahu36@gmail.com

\begin{abstract}
RESUMEN
La presente investigación es un estudio multidisciplinario que implica adecuar el trabajo a las necesidades de las personas, donde para ello se debe tener en cuenta el diseño, ya que cuenta con el riesgo asociado a la toma de posturas, la supervisión de que todo personal lleve puesto sus Equipos de Protección personal (EPP), para que así se reduzca la probabilidad de que el trabajador sufra una lesión, si es que pasa algo en el entorno laboral. El objetivo de la actual investigación es evaluar el impacto de la ergonomía para la mejora de la Productividad, una revisión sistemática entre los años 2016 - 2021., para lo cual se hizo uso de una metodología PRISMA (Preferred Reporting Items for Systematic reviews and Meta-Analyses), en cuanto a la población se tuvo en cuenta la cantidad de 66 artículos provenientes de las principales revistas científicas digitales Proquest, Science direct, Scopus entre otros, de los cuales solo se admitió a trabajar con 40 artículos como muestra debido a los requisitos establecidos en los criterios de inclusión, así como de exclusión. En conclusión, en base a los resultados hallados en esta investigación se evidencia que la ergonomía si influye en la mejora de la productividad.
\end{abstract}

Palabras clave: productividad, riesgos laborales, seguridad laboral y ergonomía. 


\begin{abstract}
The present research is a multidisciplinary study that involves adapting the work to the needs of people, where for this purpose the design must be taken into account, since it has the risk associated with the taking of postures, the supervision that all personnel wear their Personal Protective Equipment (PPE), so as to reduce the probability that the worker suffers an injury, if something happens in the work environment. The objective of the current research is to evaluate the impact of ergonomics for the improvement of Productivity, a systematic review between the years 2016 - 2021. For this purpose, a PRISMA (Preferred Reporting Items for Systematic reviews and Meta-Analyses) methodology was used, regarding the population, 66 articles from the main digital scientific journals Proquest, Science direct, Scopus, among others, of which only 40 articles were admitted to work as a sample due to the requirements established in the inclusion criteria, as well as exclusion criteria. In conclusion, based on the results found in this research, it is evident that ergonomics does influence productivity improvement.
\end{abstract}

Keywords: productivity, occupational hazards, occupational safety and ergonomics.

\section{INTRODUCCIÓN}

El trabajo de Taylor sobre la racionalización del trabajo comenzó a considerar la ergonomía a inicios del siglo XX, cuando se convirtió en una ciencia al final de la Segunda Guerra Mundial. En ese momento, en el diseño de sistemas, los ingenieros comenzaron a considerar los aspectos físicos y psicológicos de la conducta humana. Aspectos y su adaptabilidad en relación a las condiciones laborales y al entorno (Qimby, 2018). En un estudio realizado a enfermeras de la UCI en Turquía, se pudo analizar que los síntomas musculoesqueléticos son comunes en las piernas, la cintura y la espalda, y en las extremidades superiores. Este síntoma puede ocurrir en cualquier parte de nuestro cuerpo y está relacionado esencialmente con factores tisulares, como el tipo de hospital. La clase de trabajo por horarios y la frecuencia de los cambios en las horas de trabajo, no factores personales (Gallego, 2020). La prevención de accidentes laborales es muy importante para toda organización en general, además de obedecer con las normas técnicas establecidas, también brinda apoyo en mejorar las condiciones de trabajo, y fomentar el cuidado en la salud de los operarios. La determinación de peligros es fundamental, para así evitar los riesgos en las áreas laborales, de esta manera, reducir los accidentes y el absentismo, ahorrando así el tiempo de producción de la organización (Medina, 2020). Los riesgos ergonómicos no solo causan daños a los trabajadores, sino que también aumentan el costo económico de la 
empresa debido a la interrupción de las actividades de la empresa, bajas por enfermedad e incapacidad para trabajar. El principal riesgo ergonómico suele estar provocado por el uso de posturas compulsivas para realizar manualmente movimientos repetitivos (Muhammad, 2021). En la etapa actual, las organizaciones deben prestar cada vez más atención a la salud de sus empleados para que su producción sea cada vez mejor. En este caso, adaptando el trabajo a las necesidades humanas a través de varios métodos (como el análisis de la postura), la ergonomía se considera parte del proceso. La ergonomía, es una disciplina que tiene como propósito optimizar la interacción entre los trabajadores, las máquinas y el ambiente de trabajo, para que el lugar de trabajo, el entorno y la organización del trabajador se adapten a las habilidades y características del trabajador. Se analiza la ergonomía del área de oficinas, con el fin de evaluar cómo influye la ergonomía en el rendimiento de los empleados en los últimos años (Golasi, 2019).

\section{METODOLOGÍA}

La investigación actual hizo uso de una metodología PRISMA (Preferred Reporting Items for Systematic reviews and Meta-Analyses). La población está conformada por la cantidad de "66 artículos" provenientes de las principales revistas científicas digitales Ebsco, Proquest, Scielo, Science direct, Scopus, de las cuales se procedió a trabajar con 40 del total debido a los requisitos establecidos en los criterios tanto de exclusión los cuales fueron: no considerar artículos que no pertenezcan a la línea de investigación, el cual es, "Sistema de Gestión de la seguridad y calidad", asimismo que no contenga información sobre la variable ergonomía, no sean de alto impacto y reputación, que su idioma sea diferente al inglés o diferente a nuestro idioma natal (el español), que no tengan relación con la variable de estudio, y que su publicación sea anterior al año 2016. Por ello no se consideró revistas que no cuenten con un valor científico comprobado. Por otra parte, los criterios de inclusión fueron: considerar incluir artículos y revistas científicas a nivel local e internacional que pertenezcan a la ruta de indagación, "Sistema de Gestión de la seguridad y calidad", que contenga información sobre la variable ergonomía, que su fecha de publicación sea entre el rango de los años 2016 - 2021, y su idioma sea tanto en inglés como en español, y considerar revistas con un valor científico comprobado.

\section{RESULTADOS}

De los resultados observados en esta investigación científica, titulada: "Impacto de la ergonomía en la Productividad, una revisión sistemática entre los años 2016 - 2021.”, se encontró un número de variables asociadas a la ergonomía las cuales podrían desempeñar un rol importante y significativo no solo por la prevención de accidentes laborales, lo cual evidenciaría una reducción de costos, así como también un aumento de la productividad.

\section{DISCUSIÓN}

Cerca del $80 \%$ de los trabajadores padecen problemas de salud mientras que el $50 \%$ sufre de trastornos musculoesqueléticos, los beneficios de aplicar la 
ergonomía van más allá de una mejora en el confort de las personas y reducción de costos, sino que le permite una mejor postura y realizar sus actividades con mayor confort lo cual se reflejó en la calidad del trabajo y un aumento de la productividad (Priyanka, 2020). Por otra parte, estos resultados coinciden con Tirloni (2018) donde concluyó que el nivel de peligro ergonómico al que están expuestos los laboradores en el ambiente de trabajo, esto está relacionado con una vida de calidad y la eficiencia productiva; por lo tanto, que la empresa considere aumentar y mantener su capacidad productiva, dependerá del estado laboral (fisiología, seguridad e integración social) de los colaboradores. La ergonomía puede renovar la eficiencia y la seguridad en el entorno laboral, su papel en la mejora del servicio de valor agregado de los agentes de seguros profesionales y la identificación de trastornos musculo esqueléticos relacionados. Los verdaderos fines de la ergonomía son, fomentar la salud y el confort, disminuir las lesiones y aumentar la productividad de la empresa (Quimby, 2018). La adopción temprana de principios ergonómicos creará un lugar de trabajo seguro, saludable y cómodo. Si ignora el programa de aplicación o la ergonomía, dañará la seguridad y la salud de los laboradores, y será contraproducente, reduciendo así la confianza tanto física como mental de los colaboradores. Como resultado, la productividad de los trabajadores disminuye. Por lo tanto, cuando se adopten las reglas y principios de la ergonomía en el lugar de trabajo, especialmente basándose en la sabiduría local, habrá problemas de salud, seguridad, efectividad, conveniencia y eficiencia. (Thamin, 2020). La salud y la conformidad de las personas son vitales, por lo que es fundamental para su mejor desempeño en los aspectos personales, familiares, laborales y sociales. Saber más sobre el valor de la higiene y la seguridad ocupacional proporciona conocimientos y comportamientos útiles que se pueden aplicar de inmediato en cualquier lugar. Por supuesto, esta investigación científica enfatiza que los esfuerzos obvios hasta ahora han mejorado el ambiente de trabajo. (Figueroa, 2018).

\section{CONCLUSIONES}

Es importante que todas las empresas del país tengan sus propios sistemas de seguridad o adopten sistemas de seguridad, los cuales ya están en el mercado. El sistema proporcionará pautas, herramientas y controles para lograr una gestión de riesgos exitosa. La política de seguridad es el compromiso del gerente general con todos los laboradores. Entre ellos, los derechos y responsabilidades pertenecen a los supervisores y trabajadores de la organización. Base a medidas de seguridad, el proceso de cambio se ha establecido y debe recibir una formación continua para avanzar, porque estos procesos son fundamentales para concienciar y mejorar la cultura de seguridad. de los colaboradores. Es algo primordial que los laboradores deban contar con el Reglamento General, aplicando de forma más clara y sencilla las normas técnicas y la ergonomía, para así poder lograr los propósitos de productividad y garantizar el confort y la salubridad del trabajador. 


\section{REFERENCIAS}

[1] Gallego, Y. (2020). Prevención de Trastornos Musculoesqueléticos mediante la mejora de Hábitos Posturales. Prevención de Riesgos Laborales, 23(2), https://dialnet.unirioja.es/servlet/articulo?co $\operatorname{digo}=7340598$

[2] Golasi, I. (2019). Forced postures in courgette greenhouse workers. International Journal of Environmental Research and Public Health, 9(5). https://bit.ly/3C6w3Vb

[3] Medina, E. (2020). Evaluation of disergonomic risks in small and medium-size enterprises (SMEs) in Bogotá. Universidad Nacional de Colombia, (DYNA), 14(1). https://bit.ly/3wHNWse

[4] Muhammad, B. (2021). Upper extremity musculoskeletal disorders and exposure to Ergonomic risk factors among handicraft workers. Pakistan Journal of Medical Sciences Quarterly, 37(2). https://www.proquest.com/docview/251275 6834/abstract/66446A832354A7EPQ/1?acc ountid $=37408$

[5] Priyanka, K. (2020). Occupational health problems of the handloom workers: A cross sectional study of Sualkuchi, Assam, Northeast India. Clinical Epidemiology and Global Health, 8(4). https://bit.ly/3oiLC72

[6] Quimby, S. (2018). Ergonomics - Increasing Safety and Efficiency. Insurance Advocate, 129(16). https://bit.ly/3Dez4nv
[7] Thamin, Y. (2020). Ergonomics and musculoskeletal disorders among seaweed workers in Takalar Regency: A mixed method approach. Medicina Clínica Práctica, 3(1).

https://doi.org/10.1016/j.mcpsp.2020.10011 0 